\title{
Sulfur dioxide reduces lipopolysaccharide- induced acute lung injury in rats
}

\author{
YU ZHAI ${ }^{*}$, XIN-LI HUANG ${ }^{2 *}$, HUI-JIE MA ${ }^{3}$, XIAO-HONG ZHOU ${ }^{1}$, JUN-LIN ZHOU ${ }^{4}$, YA-MIN FAN ${ }^{2}$ \\ ${ }^{1}$ Department of Basic Medicine, Hebei University of Chinese Medicine, Shijiazhuang, China \\ ${ }^{2}$ Department of Pathophysiology, Hebei Medical University, Shijiazhuang, China \\ ${ }^{3}$ Department of Physiology, Hebei Medical University, Shijiazhuang, China \\ ${ }^{4}$ Department of Orthopedics, Beijing Chaoyang Hospital, Capital Medical University, Beijing, China \\ "These authors contributed equally to this work.
}

\begin{abstract}
Introduction: Recent studies suggested that sulfur dioxide (SO2) can be produced endogenously by pulmonary vessels and attenuate acute lung injury (ALI) with vasorelaxant effects. This study was conducted to determine whether SO2 can inhibit lung inflammation and relax pulmonary arteries via inhibition of the mitogen-activated protein kinase (MAPK) pathway.

Material and methods: Forty-eight adult male Sprague Dawley rats (250 300 g) were randomly divided into six treatment groups: control $(n=8)$, control $+S O 2(n=8)$, control + L-aspartic acid- $\beta$-hydroxamate $(H D X)(n=8), L P S(n=8), L P S+S O 2(n=8)$ and LPS + HDX $(n=8)$.

Results: Six hours after LPS treatment, rats exhibited elevated pulmonary artery hypertension $(P A H)$, marked pulmonary structure injury with elevated pulmonary myeloperoxidase (MPO) activity and increased expression of intercellular adhesion molecule 1 (ICAM-1) and CD11b, along with decreased pulmonary SO2 production and reduced pulmonary aspartate aminotransferase (AAT) activity. Pretreatment with SO2 saline solution significantly reduced, while HDX (AAT inhibitor) aggravated, the pathogenesis of LPS-induced ALI. Moreover, SO2 saline solution significantly down-regulated expression of Raf-1, MEK-1 and phosphorylated ERK ( $p$-ERK). It also prevented pulmonary hypertension in association with an up-regulated SO2/AAT pathway. However, HDX advanced pulmonary hypertension and inflammatory responses in the lung were associated with a down-regulated SO2/AAT pathway.

Conclusions: Our results suggest that $\mathrm{SO} 2$ markedly relieved inflammatory responses, in association with Raf-1, MEK-1 and p-ERK during ALI induced by LPS. The down-regulation of the SO2/AAT pathway may be involved in the mechanism(s) of LPS-induced lung injury.
\end{abstract}

Key words: sulfur dioxide, acute lung injury, interleukin, inflammation.

(Centr Eur J Immunol 2019; 44 (3): 226-236)

\section{Introduction}

Endotoxin-induced acute lung injury (ALI) and its more severe form, acute respiratory distress syndrome (ARDS), are consequences of acute respiratory failure. Although the mortality rate associated with this syndrome has declined recently, ARDS remains one of the major causes of pulmonary and non-pulmonary morbidity in patients after hospital discharge [1,2], and the mechanism for ALI has not been fully established. Studies in recent years have shown that the accumulation of inflammatory cell polymorphonuclear neutrophils (PMNs) in lung tissue [3], which is mediated through integrins such as CD11b and its ligand intercellular adhesion molecule-1 (ICAM-1), is the key pathological basis of endotoxin-induced ALI. Excessive infiltration of PMNs in response to infection causes an imbalance between inflammatory and anti- inflammatory cytokines [3-5], as well as abnormal vascular reactivity in the pulmonary circulation $[6,7]$, which further stimulate immune responses and ultimately promote the development of ALI.

Gaseous messenger molecules (signal transmitters), including nitric oxide (NO), carbon monoxide (CO) and hydrogen sulfide $\left(\mathrm{H}_{2} \mathrm{~S}\right)$, played important roles in both physiological and pathological conditions, including those of the inflammatory response [8-11]. In recent years, interest has been directed towards another naturally occurring gas, sulfur dioxide $\left(\mathrm{SO}_{2}\right)$. $\mathrm{SO}_{2}$ is a common gaseous pollutant in the atmosphere, generally regarded as an environmental toxin. Epidemiological investigations have found that $\mathrm{SO}_{2}$ exposure correlated with development of cardiovascular diseases in humans [12]. In addition, experimental studies have revealed that $\mathrm{SO}_{2}$ and its derivatives, sulfite and bisulfite, could act as systemic toxic substances, capable

Correspondence: Xiao-Hong Zhou, PhD, Department of Basic Medicine, Hebei University of Chinese Medicine, 050091 Shijiazhuang,

China, e-mail: xiao_hong0217@sina.com

Submitted: 19.02.2017; Accepted: 19.04.2017 
of affecting multiple organs in mammals [13-16]. Endogenous $\mathrm{SO}_{2}$ can be produced during the normal processing of sulfur-containing amino acids such as L-cysteine in vivo, which is first oxidized to L-cysteine sulfinate, and the latter can develop through transamination by aspartate aminotransferase into $\beta$-sulfinylpyruvate, which decomposes spontaneously to pyruvate and $\mathrm{SO}_{2}$, and it had been shown that the $\mathrm{SO}_{2}$ /aspartate aminotransferase (AAT) pathway could regulate vascular activities and was involved in the inflammatory response in vascular and lung tissues [17, 18]. In addition, our previous study suggested that $\mathrm{SO}_{2}$ attenuates LPS-induced ALI via enhancing apoptosis of PMNs [19]. So, in this study, we decided to investigate and validate the hypothesis that endogenous gaseous $\mathrm{SO}_{2}$ may also act as a messenger molecule that could regulate inflammatory responses and vascular reactivity of pulmonary arteries (PAs), which are particularly involved in ALI.

\section{Material and methods}

\section{Preparation of experimental animal model}

All experiments were approved by the Animal Ethics Committee of the National University of China (No. 0126), and all experiments were carried out in accordance with established guiding principles for animal research. Forty-eight adult male Sprague Dawley rats (250 300 g) were randomly divided into six treatment groups: control $(n=8)$, control $+\mathrm{SO}_{2}(n=8)$, control $+_{\mathrm{L}}$-aspartic acid- $\beta$-hydroxamate (HDX) $(n=8)$, LPS $(n=8), \mathrm{LPS}+\mathrm{SO}_{2}$ $(n=8)$ and LPS $+\operatorname{HDX}(n=8)$. For this experiment, rats were anesthetized with ether inhalation, which did not influence the patterns of inflammation and $\mathrm{SO}_{2}$ production (data not shown). Control rats were instilled intratracheally with $200 \mu \mathrm{l}$ of normal saline, while LPS-treated animals were instilled intratracheally with $200 \mu \mathrm{l}$ of LPS (Escherichia coli $055: \mathrm{B} 5 ; 100 \mu \mathrm{g} / 100 \mathrm{~g}$ of body weight, dissolved in $200 \mu \mathrm{l}$ of saline, Sigma, St. Louis, MO, USA). HDX, an inhibitor of endogenous $\mathrm{SO}_{2}$ generating enzymes, was injected intraperitoneally (i.p., $3.7 \mathrm{mg} / \mathrm{kg}$, Sigma) into rats in the LPS + HDX group 30 min before LPS instillation. The HDX dose used was based on observations of $\mathrm{SO}_{2}$-independent effects in a previous study [20] and our own preliminary experiment. The same dosage of HDX was injected i.p. into rats of the control + HDX group. For rats in the control $+\mathrm{SO}_{2}$ group and LPS $+\mathrm{SO}_{2}$ group, $\mathrm{SO}_{2}$ saline solution $(25 \mu \mathrm{mol} / \mathrm{kg})$ was injected i.p. $30 \mathrm{~min}$ before saline or LPS instillation. The concentration of $\mathrm{SO}_{2}$ also was based on a previous study [20] and our preliminary experiment. An identical volume of saline was injected into rats of the control group and LPS group.

The $\mathrm{SO}_{2}$ solution was freshly prepared before each experiment by bubbling saline with pure $\mathrm{SO}_{2}$ gas (purity: 99.99\%, Beijing He-Pu-Bei-Fen Gas Company, Ltd., Beijing, China) and diluting it to achieve $\mathrm{SO}_{2}$ concentrations required according to the previous method [21]. Six hours after the LPS instillation, animals were sacrificed by an i.p. injection of a lethal dose of pentobarbitone $(90 \mathrm{mg} / \mathrm{kg}$ ). Blood samples were taken from the right ventricles using heparinized syringes and then centrifuged $(4000 \times \mathrm{g}$, $10 \mathrm{~min}, 0-4^{\circ} \mathrm{C}$ ). Thereafter, plasma and lung tissue specimens were stored at $-80^{\circ} \mathrm{C}$ for subsequent measurement of $\mathrm{SO}_{2}$ concentrations.

\section{Measurement of pulmonary arterial pressure}

Six hours after the LPS instillation, rats were anesthetized with pentobarbitone $(50 \mathrm{mg} / \mathrm{kg}$, i.p. $)$. A silicone catheter (outer diameter, $0.9 \mathrm{~mm}$ ) was introduced into the right jugular vein by venotomy. It was passed across the tricuspid valve and right ventricle into the PA. The distal end of the catheter was connected to a PowerLab system via a pressure transducer (model Gould P23Db, AD Instruments, Sidney, Australia) for pulmonary arterial pressure (PAP) measurements.

\section{Determination of PA reactivity}

After PAP measurements, the PAs were rapidly isolated and placed in a $4^{\circ} \mathrm{C}$ modified Krebs-Henseleit (K-H) solution containing the following (in $\mathrm{mM}$ ): $\mathrm{NaCl}$ 118.0, $\mathrm{KCl}$ 4.7, $\mathrm{CaCl}_{2}$ 2.5, $\mathrm{MgSO}_{4} 1.2, \mathrm{NaHCO}_{3} 25.0, \mathrm{KH}_{2} \mathrm{PO}_{4}$ 1.2 and glucose 11.0, gassed with $95 \% \mathrm{O}_{2}$ and $5 \% \mathrm{CO}_{2}$ ( $\mathrm{pH}$ 7.2-7.4). The PA adventitia was cleared and cut into 3 -mm width rings. PA rings were mounted in organ chambers filled with $6 \mathrm{ml} \mathrm{K-H}$ solution (gassed with $95 \% \mathrm{O}_{2}$ and $5 \% \mathrm{CO}_{2}, 37 \pm 0.5^{\circ} \mathrm{C}, \mathrm{pH} 7.4$ ), which was supplemented with $10^{-5} \mathrm{~mol} / \mathrm{l}$ indomethacin. At the beginning of the experiment, each PA ring was stretched to its optimal resting tension, which was achieved stepwise until the active contraction of the PA ring in response to $100 \mathrm{mM} \mathrm{KCl}$ reached a plateau. The optimal resting tension of the rat PAs was approximately $1.0 \mathrm{~g}$. The vessels were allowed to equilibrate for $1 \mathrm{~h}$, and the K-H solution was changed every $15 \mathrm{~min}$. The contractile responses were performed by cumulative addition of phenylephrine (PE, $10^{-8}-10^{-5} \mathrm{M}$, Sigma) and expressed as a percentage of the response to $100 \mathrm{mM} \mathrm{KCl}$. The viability and integrity of the arterial endothelium were estimated by the analysis of the relaxant response to cumulative addition of acetylcholine (ACh, 10${ }^{8}-10^{-5} \mathrm{M}$, Sigma) or sodium nitroprusside (SNP, $10^{-9}-10^{-6} \mathrm{M}$, Sigma) after precontraction with $10^{-6} \mathrm{M}$ PE.

\section{Preparation and morphometric analysis of lung tissue}

After the PAP was measured, the thoracic cavity was opened. One of the lung lobes was removed and fixed in $4 \%$ (wt/vol) paraformaldehyde at $30 \mathrm{~cm} \mathrm{H}_{2} \mathrm{O}$ pressure. After routine processing and paraffin embedding, lung tissues were sliced into thin sections (5- $\mu \mathrm{m}$ thickness) and stained with hematoxylin and eosin (H\&E). Based on the degree of cellular and protein exudation and tissue hemorrhage, 
the extent of lung injury was determined by assigning a score between 0 (normal) and 3 (severely damaged) for each parameter as previously reported [22]. The severity of lung injury was scored by two pulmonologists, who were blinded to the treatments. Ten fields were randomly selected from three lung tissue sections per rat and examined under a microscope at 200× magnification. The degree of cellular and protein exudation and tissue hemorrhage per rat was determined by adding the scores from 10 randomly selected fields. The total lung injury score per rat was determined by the sum of three individual scores for alveolar cellular and protein exudation and tissue hemorrhage.

\section{Determination of myeloperoxidase activity in lung tissue}

Tissue samples were homogenized in $20 \mathrm{mM}$ phosphate buffer $(\mathrm{pH} 7.4)$ and centrifuged $(13,000 \times \mathrm{g}$, $10 \mathrm{~min}, 4^{\circ} \mathrm{C}$ ). The resulting pellets were resuspended in $50 \mathrm{mM}$ phosphate buffer ( $\mathrm{pH}$ 6.0) containing $0.5 \%$ (wt/vol) hexadecyltrimethylammonium bromide (Sigma). The suspension was then subjected to four cycles of freezing and thawing and further disrupted by sonication (40 s). The samples were then centrifuged $\left(13,000 \times \mathrm{g}, 5 \mathrm{~min}, 4^{\circ} \mathrm{C}\right)$, and the supernatants were collected for immediate use in the myeloperoxidase (MPO) assay. The MPO reaction mixture consisted of the supernatant $(50 \mu \mathrm{l}), 1.6 \mathrm{mM}$ tetramethylbenzidine (Sigma), $80 \mathrm{mM}$ sodium phosphate buffer (pH 5.4) and $0.3 \mathrm{mM}$ hydrogen peroxide. This mixture was incubated at $37^{\circ} \mathrm{C}$ for $110 \mathrm{~s}$, and the reaction was terminated by addition of $50 \mu \mathrm{l}$ of $0.18 \mathrm{M} \mathrm{H}_{2} \mathrm{SO}_{4}$. The absorbance was measured at $405 \mathrm{~nm}$ and normalized by the DNA content of the original tissue sample [23]. Results were expressed as the fold change in MPO activity in the test sample over that of the control.

\section{Measurements of IL-1 $\beta$, IL-6 and IL-10 levels in lung tissue}

Six hours after the LPS instillation, the lower lobes of the right lung were homogenized and centrifuged. The supernatants were collected for testing IL- $1 \beta$, IL- 6 and IL-10 concentrations. Blood samples ( $4 \mathrm{ml}$ from each rat) from each group were collected in heparinized tubes by jugular vein catheterization and centrifuged $(3,000 \times \mathrm{g}, 10 \mathrm{~min})$. Cytokines levels were assayed using a double-antibody sandwich enzyme-linked immunosorbent assay (ELISA) following the manufacturer's instructions (Diaclone, Fleming, France). Briefly, samples or standards for IL-1 $\beta$, IL-6 or IL-10 were added in duplicate to the ELISA plate in $100 \mu \mathrm{l}$ per well. After incubation at $37^{\circ} \mathrm{C}$ for $1 \mathrm{~h}$, samples were removed, and the plates were washed five times with a wash buffer (10 mmol/1 PBS, pH 7.4, and 0.1\% Tween-20). Blotted plates were dried by tapping upside down on filter paper. Biotinylated anti-rat IL-1 $\beta$, IL-6 or IL-10 (100 $\mu \mathrm{l})$ was added to each well of the plates, which were incubated for $30 \mathrm{~min}$ at $37^{\circ} \mathrm{C}$. After five additional washes, $100 \mu \mathrm{l}$ of horseradish peroxidase (HRP) was added to each well, and the plates were incubated for another $30 \mathrm{~min}$ at $37^{\circ} \mathrm{C}$. Following a final series of five washes, $100 \mu$ l of tetramethylbenzidine (TMB) substrate was added to each well with gentle shaking for $10 \mathrm{~s}$. The mixture was then incubated in the dark for $30 \mathrm{~min}$ at room temperature. The reaction was terminated by adding $100 \mu$ l of stop solution to each well, and the optical density (OD) at $450 \mathrm{~nm}$ was measured with an ELISA reader (Bio-Rad, Hercules, CA, USA). Cytokine concentrations were obtained by plotting sample OD values against those of the corresponding standard curve.

\section{Determination of $\mathrm{SO}_{2}$ content in lung tissue}

The right lung samples were homogenized and immediately processed for analysis. Each homogenate $(100 \mu \mathrm{l})$ was mixed with $70 \mu \mathrm{l}$ of $0.212 \mathrm{mmol} / \mathrm{l}$ sodium borohydride in $0.05 \mathrm{~mol} / \mathrm{l}$ Tris- $\mathrm{HCl}(\mathrm{pH} 8.5)$ and incubated at room temperature for $30 \mathrm{~min}$. The samples were then mixed with $10 \mu \mathrm{l}$ of $70 \mathrm{mmol} / \mathrm{l}$ monobromobimane in acetonitrile. After incubation for $10 \mathrm{~min}$ at $42^{\circ} \mathrm{C}, 40 \mu \mathrm{l}$ of $1.5 \mathrm{mmol} / \mathrm{l}$ perchloric acid solution was added to the mixture, followed by vortex mixing. The protein precipitates were removed by centrifugation at $12,400 \times \mathrm{g}$ for $10 \mathrm{~min}$ at room temperature. The supernatant was immediately neutralized

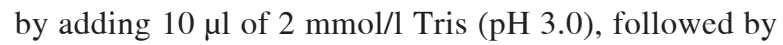
gentle mixing and centrifugation again at $12,400 \times \mathrm{g}$ for $10 \mathrm{~min}$. Ten microliters of the neutralized supernatant was injected onto a high performance liquid chromatography (HPLC) column (Hitachi 655A-11 HPLC system, Tokyo, Japan). All procedures of the tissue sample preparation were completed within $2 \mathrm{~h}$ after homogenization. Samples were resolved on a $4 \times 250 \mathrm{~mm} \mathrm{C} 8$ reversed phase column (5 $\mu$ m packing, GL Science, Tokyo, Japan). The column was equilibrated with methanol/acetic acid/water ( $5.00: 0.25: 94.75$, by volume, $\mathrm{pH} 3.4$ ) and developed with a gradient of methanol at a flow rate of $0.8 \mathrm{ml} / \mathrm{min}$ as follows: 0-5 min, $30 \mathrm{ml} / 1$; 5-13 $\mathrm{min}, 30-500 \mathrm{ml} / 1$; $13-30 \mathrm{~min}$, 350-620 ml/1; 30-31 min, 620-1000 ml/1; 31-39 min, $1000 \mathrm{ml} / 1$; 39-40 $\mathrm{min}, 1000-30 \mathrm{ml} / \mathrm{l}$; and 40-46 min, $30 \mathrm{ml} / \mathrm{l}$. Sulfite-bimane was detected by excitation at $390 \mathrm{~nm}$ and emission at $472 \mathrm{~nm}$ using a cut-off filter and eluted at the methanol concentration of $45 \mathrm{ml} / \mathrm{l}$ [24].

\section{Determination of AAT activity in lung tissue}

AAT activity in lung tissues was determined using the UniCel DxC 800 Synchron Clinical System (Beckman Coulter, Inc., Fullerton, CA, USA). The lung tissues of rats were homogenized with $0.01 \mathrm{~mol} / \mathrm{l}$ PBS (weight : volume, $1: 10$ ). Protein precipitates were removed by centrifugation at $2,500 \times \mathrm{g}$ for $10 \mathrm{~min}$ at room temperature. The supernatant was used to detect AAT activity by the Reitman method, and tissue proteins were analyzed by Coomassie Blue staining. 


\section{Western blotting analysis}

After homogenization and lysing of rat lung tissues, equal amounts of proteins were boiled, separated using sodium dodecyl sulfate polyacrylamide gel electrophoresis (SDS-PAGE) and electrotransferred to a polyvinylidene difluoride (PVDF) membrane. Non-specific antibody binding was blocked by washing with Tris-buffered saline (TBS) containing 5\% non-fat milk for $1 \mathrm{~h}$. The membranes were then washed twice with PBST (0.01 M PBS mixed with $0.1 \%$ Tween-20) and incubated with the primary antibody for $1 \mathrm{~h}$. The following primary antibodies were used (all from Santa Cruz unless otherwise specified): rabbit antibodies to ICAM-1 (1 : 1,000 dilution), CD11b (1 : 1,000 dilution), ERK (1 : 1,000 dilution), phosphorylated ERK (p-ERK, $1: 2,00$ dilution), Raf-1 (1 : 2,00 dilution) and MEK-1 ( $1: 2,00$ dilution); and a mouse antibody to $\beta$-actin (1:10,000, Abcam, Cambridge, UK). After washing with PBST, the membranes were incubated with biotin-labeled anti-rabbit IgG for ICAM-1, CD11b, ERK, p-ERK, Raf-1 or MEK-1 (diluted 1 : 4,000 in 0.01 M PBS, Chemicon, Billerica, MA, USA) and biotin-labeled anti-mouse IgG for $\beta$-actin (diluted $1: 4,000$ in $0.01 \mathrm{M}$ PBS, KPL, Gaithersburg, MD, USA) for $1 \mathrm{~h}$ at $37^{\circ} \mathrm{C}$. After washing with PBST again, the filters were incubated with HRP-conjugated streptavidin (diluted $1:$ 4,000 in $0.01 \mathrm{M}$ PBS, Zymed, Carlsbad, CA, USA) for $1 \mathrm{~h}$ at $37^{\circ} \mathrm{C}$. The immunoreactions were visualized using an ECL detection kit (Pierce, Thermo Scientific, Waltham, MA, USA). The X-ray film was scanned using an image documentation system (Labworks Inc., Costa Mesa, CA, USA). The integral optical density (IOD) of each band was measured using the AlphaImager 2200 gel image analyzing system (Genetic Technologies, Miami, FL USA). Changes in the relative content of ICAM-1, CD11b, ERK, p-ERK, Raf-1 and MEK-1 protein were represented by the ratio of the IOD of each protein band to that of $\beta$-actin.

\section{Statistical analysis}

All data were calculated as means $\pm \mathrm{SD}$. Comparisons between groups were performed using analysis of variance (ANOVA), followed by the Student-Newman-Keuls test, using the SPSS 13.0 statistical analysis software. Statistical significance was accepted when $p<0.05$. The optical density data from the Western blotting analysis were determined to be normally distributed at the $\alpha$ level of 0.05 and with the statistical power of 0.9 .

\section{Results}

\section{Induction of ALI is associated with decrease in $\mathrm{SO}_{2}$ concentration}

LPS-induced lung injury is indicated by MPO activity, a marker of tissue neutrophil infiltration, and histological changes as well as cytokine changes. As expected, lung MPO activity ( $p<0.01$, Fig. 1 ) and the IL-1 $\beta$, IL-6 and IL-10 protein levels were significantly elevated after LPS treatment ( $p<0.01$, Fig. 2A-2C), compared with the control group. Characteristic morphological changes in the lung also suggested inflammatory damage. The lung sections from rats with ALI induced by LPS exhibited interstitial edema, alveolar thickening and severe leukocyte infiltration in the interstitium and alveoli. Therefore, the total lung injury score was elevated significantly by LPS treatment $(p<0.01$, Fig. 3). Furthermore, the mean PAP of the ALI rat group was increased significantly after LPS treatment ( $p<0.01$, Fig. 4). Moreover, LPS-induced lung injury was accompanied by significantly reduced AAT activity $\left(p<0.05\right.$, Fig. 5A) and $\mathrm{SO}_{2}$ concentration $(p<0.05$, Fig. 5B) in the lung, compared with the control group. In addition, compared with control rats, administration of HDX $(3.7 \mathrm{mg} / \mathrm{kg}) 30 \mathrm{~min}$ before saline intratracheal instillation decreased AAT activity ( $p<0.05$, Fig. 5A), which caused a significant reduction in $\mathrm{SO}_{2}$ concentration in the rat lung $(p<0.01$, Fig. 5B). Meanwhile the lung MPO activity $(p<0.05$, Fig. 1), lung inflammatory response $(p<0.01$, Fig. 2A-2C) and mean PAP ( $p<0.05$, Fig. 4$)$ were all significantly higher than those of rats intratracheally instilled with saline. HDX administration also produced histological evidence of lung injury, resulting in a significantly higher total lung injury score relative to the control $(p<0.01$, Fig. 3).

\section{Treatment with $\mathrm{HDX}$ attenuates $\mathrm{SO}_{2}$ formation and aggravates lung injury induced by LPS}

Administration of HDX $(3.7 \mathrm{mg} / \mathrm{kg}) 30 \mathrm{~min}$ before the LPS intratracheal instillation decreased AAT activity $(p<0.05$, Fig. 5A) and caused a significant reduction in $\mathrm{SO}_{2}$ concentration in the lungs of rats with $\mathrm{ALI}(p<0.01$, Fig. 5B). Endogenous $\mathrm{SO}_{2}$ formation blocked by $\mathrm{HDX}$ injection also caused a further and pronounced rise in lung

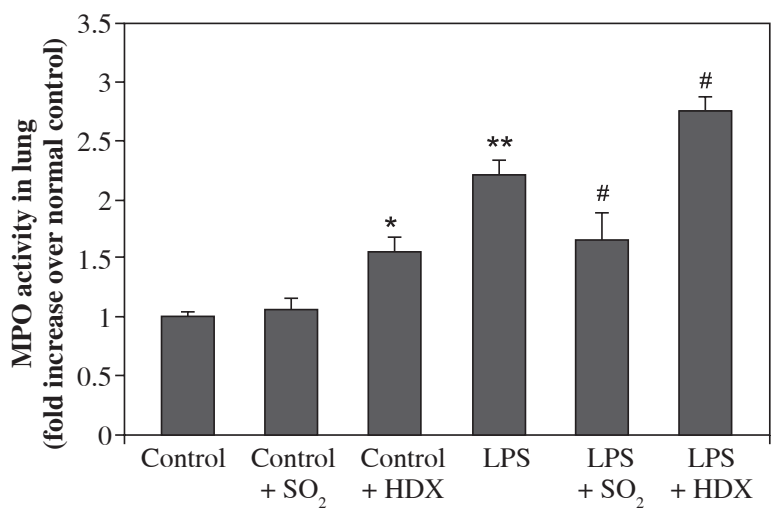

Fig. 1. Effects of $\mathrm{SO}_{2}$ on myeloperoxidase (MPO) activity in rat lung tissues. Data are presented as the mean $\pm \mathrm{SD}$ ( $n=8$ in each group); ${ }^{*} p<0.05, * * p<0.01$, compared with control group, \# $p<0.05$, compared with LPS group 
A

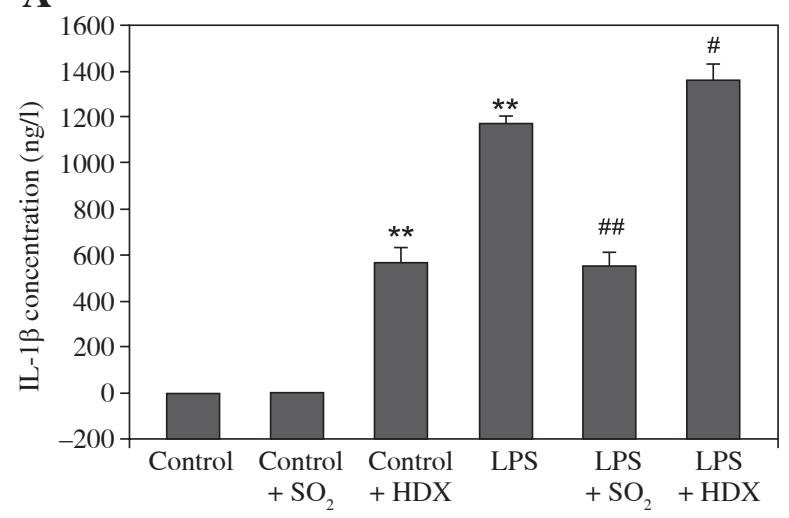

C
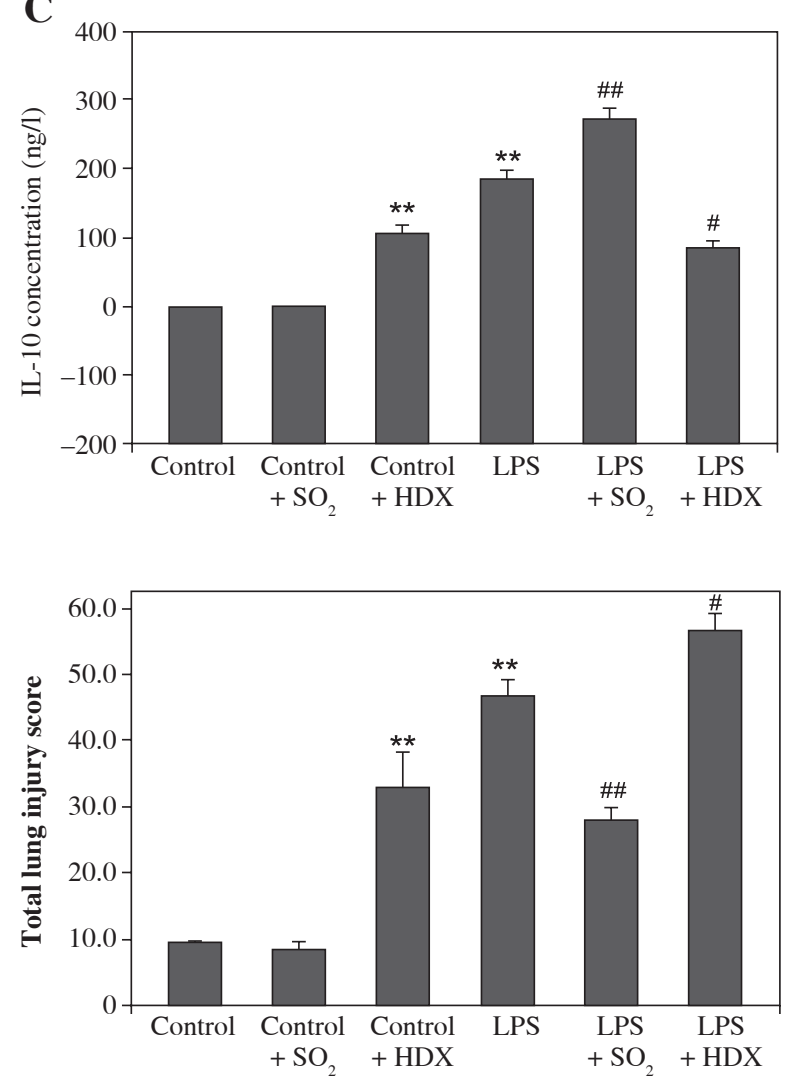

Fig. 3. Total lung injury score of rats in different groups. Data are presented as the mean $\pm \mathrm{SD}(\mathrm{n}=8$ in each group); $* * p<0.01$, compared with control group, \# $p<0.05$, $\# \# p<0.01$, compared with LPS group

MPO activity in the LPS-induced injured lung compared with the effect of saline injection $(p<0.05$, Fig. 1). Pretreatment with HDX before LPS significantly aggravated lung inflammatory responses, as evidenced by significantly increased IL- $1 \beta$ and IL- 6 protein levels and a decreased IL-10 protein level in lung tissues $(p<0.05$ or $p<0.01$, Fig. 2A-2C). Moreover, the HDX administration also produced histological evidence of more severe lung injury

\section{B}

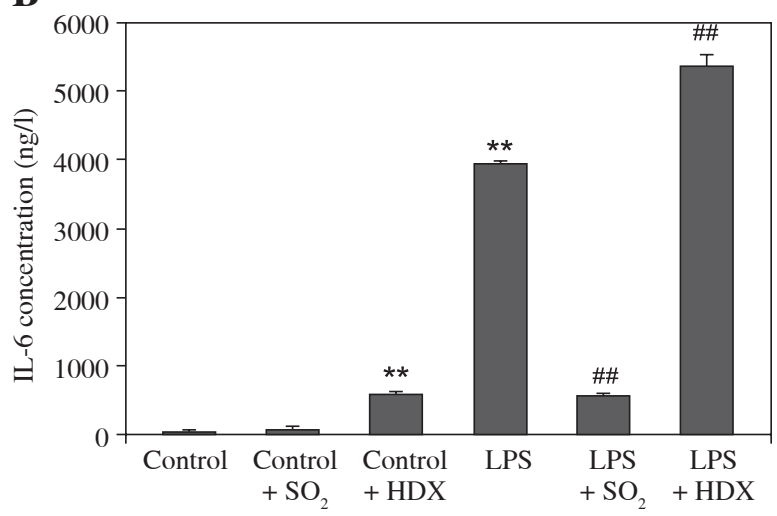

Fig. 2. IL-1 $\beta$ (A), IL-6 (B) and IL-10 (C) concentrations in rat lung tissues determined by ELISA. Data are presented as the mean $\pm \mathrm{SD}$ ( $n=8$ in each group); ** $p<0.01$, compared with control group, \# $p<0.05$, \#\# $p<0.01$, compared with LPS group

and, therefore, a significantly higher total lung injury score resulting from the increased pulmonary congestion, red blood cells, leukocyte infiltration and alveolar wall thickness ( $p<0.05$, compared with saline injection; Fig. 3 ). In addition, administration of HDX also significantly increased the mean PAP of the ALI rat group relative to that of the saline injected group $(p<0.01$, Fig. 4).

\section{Treatment with $\mathrm{SO}_{2}$ saline solution attenuates LPS-induced lung injury}

To directly investigate the potential anti-inflammatory role of $\mathrm{SO}_{2}$ in ALI, a $25 \mu \mathrm{mol} / \mathrm{kg} \mathrm{SO}_{2}$ saline solution was injected into the animals $30 \mathrm{~min}$ before LPS instillation. We found that $\mathrm{SO}_{2}$ pretreatment in both control rats and ALI rats resulted in a significant increase in $\mathrm{SO}_{2}$ concentrations in the lung ( $p<0.05$ or $p<0.01$, Fig. $5 \mathrm{~B}$ ), while the AAT activity in the lung did not change $(p>0.05$, Fig. 5A). Administration of the $\mathrm{SO}_{2}$ saline solution mitigated the inflammation and damage caused by LPS in the lung. Compared with saline-injected rats, exogenous $\mathrm{SO}_{2}$ from the injected $\mathrm{SO}_{2}$ saline solution caused a pronounced decrease in MPO activity in the lung at $6 \mathrm{~h}$ after LPS intratracheal instillation ( $p<0.05$, Fig. 1$)$. We also found that injection of the $\mathrm{SO}_{2}$ saline solution $30 \mathrm{~min}$ before LPS instillation led to significant decreases in IL- $1 \beta$ and IL-6 levels, but an increase in the IL-10 level in lung tissues 
$\left(p<0.01\right.$, Fig. 2A-2C). Furthermore, administration of $\mathrm{SO}_{2}$ saline solution attenuated the histological damage in the lung that was induced by LPS, consequently significantly lowering the total lung injury score $(p<0.01$, compared with saline injection; Fig. 3). Moreover, compared with the LPS group, the mean PAP was lower in the LPS $+\mathrm{SO}_{2}$ group ( $p<0.05$, Fig. 4$)$. In addition, no statistically significant changes were found between the control $+\mathrm{SO}_{2}$ group and the control group ( $p>0.05$, Figs. 1-4).

\section{ICAM-1 and CD11b protein expression in lung tissue by Western blotting}

Expression levels of ICAM-1 and CD11b proteins in the lung tissues of rats in the LPS group were both higher than those in the control group (both $p<0.01$, Fig. 6). In the $\mathrm{LPS}+\mathrm{SO}_{2}$ group, the levels of both ICAM-1 and CD11b decreased when compared with the LPS group $(p<0.05$ or $p<0.01$, respectively, Fig. 6). However, when treated with HDX, the rats showed increased expression of ICAM-1 and CD11b proteins in the lung tissue of the LPS group (both $p<0.01$, Fig. 6); meanwhile, these proteins did not change in the control $+\mathrm{SO}_{2}$ group ( $p>0.05$, Fig. 6) but were elevated in the control + HDX group compared with the control group ( $p<0.05$ or $p<0.01$, respectively, Fig. 6).

\section{Raf-1 and MEK-1 expression in lung tissue by Western blotting}

Compared with the control group, the expression of Raf- 1 and MEK- 1 proteins in the LPS group rats clearly rose ( $p<0.05$ and $p<0.01$, respectively, Fig. 7); however, these proteins in rats of the LPS $+\mathrm{SO}_{2}$ group decreased compared with the LPS group $(p<0.05$ and $p<0.01$, respectively, Fig. 7). The expression of Raf-1 and MEK-1 proteins did not change in the control $+\mathrm{SO}_{2}$ group $(p>0.05$, Fig. 7$)$, while it increased significantly in the

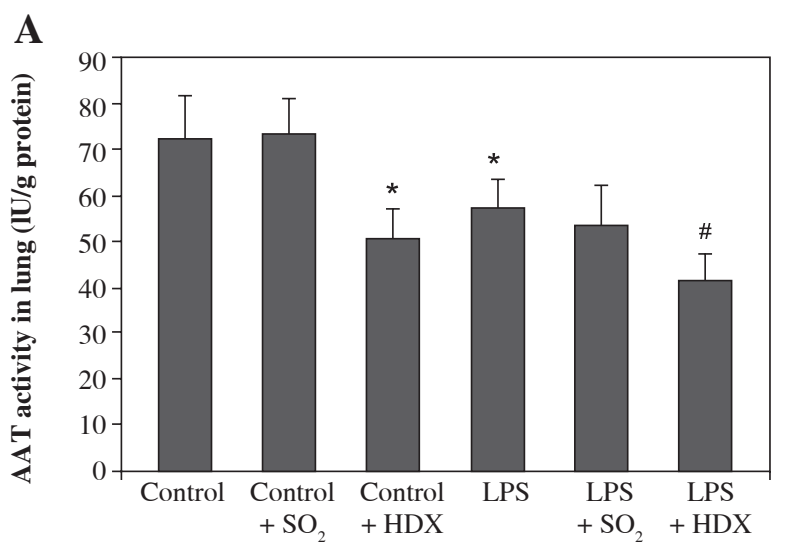

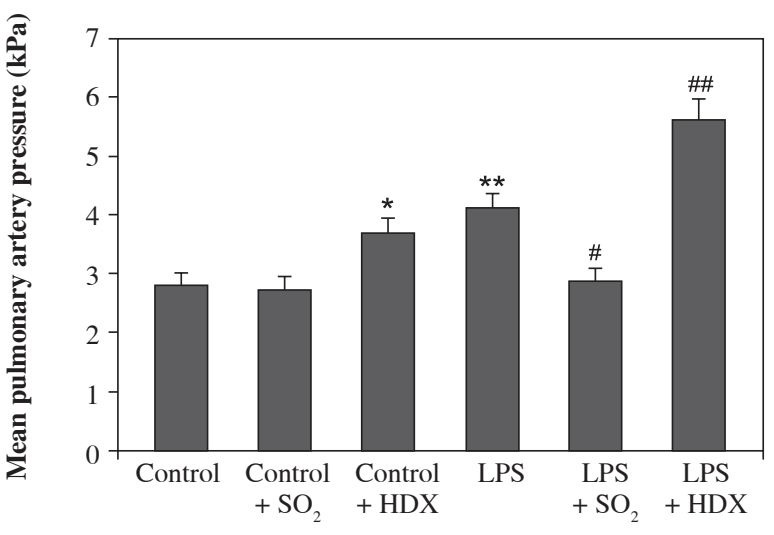

Fig. 4. Mean pulmonary artery pressure (mPAP) of rats. Data are presented as the mean $\pm \mathrm{SD}$ ( $n=8$ in each group); $* p<0.05, * * p<0.01$, compared with control group, $\# p<0.05$, \#\# $p<0.01$, compared with LPS group

control + HDX group compared with the control $(p<0.05$ and $p<0.01$, respectively, Fig. 7).

\section{Expression of p-ERK/ERK in lung tissue by Western blotting}

Compared with the control group, expression of p-ERK in rats of the LPS group clearly increased $(p<0.01$, Fig. 8). Compared with the LPS group, p-ERK expression in rats of the LPS $+\mathrm{SO}_{2}$ group decreased ( $p<0.01$, Fig. 8). $\mathrm{SO}_{2}$ did not alter, while HDX, as an inhibitor of endogenous production of $\mathrm{SO}_{2}$, significantly increased the expression of $\mathrm{p}$-ERK protein in rats undergoing endotracheal instillation with normal saline ( $p<0.05$, Fig. 8$)$. There was no significant difference in ERK expression in the lung of rats among the six groups ( $p>0.05$, Fig. 8$)$.

\section{B}

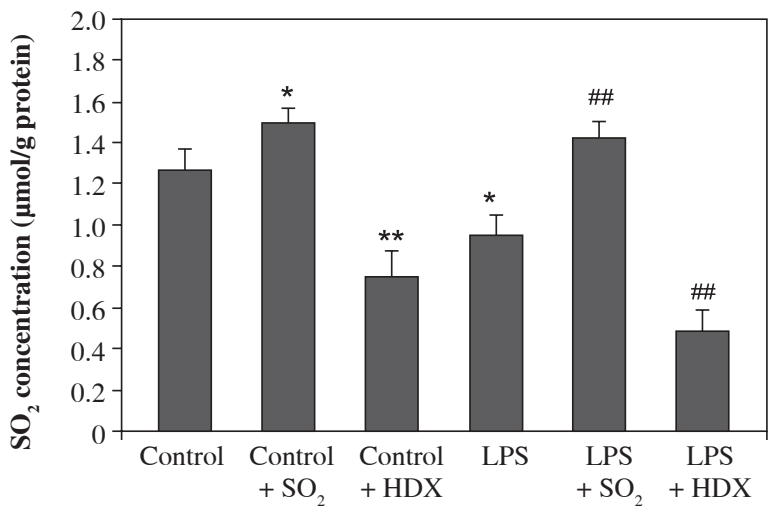

Fig. 5. AAT activity $(\mathbf{A})$ and $\mathrm{SO}_{2}$ content $(\mathbf{B})$ in rat lung tissues. Data are presented as the mean $\pm \mathrm{SD}(n=8$ in each group); * $p<0.05, * * p<0.01$, compared with control group, \# $p<0.05$, \#\# $p<0.01$, compared with LPS group 
A

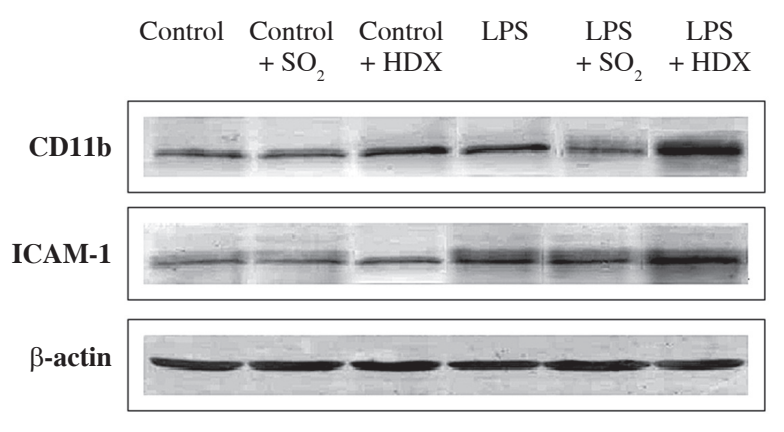

B

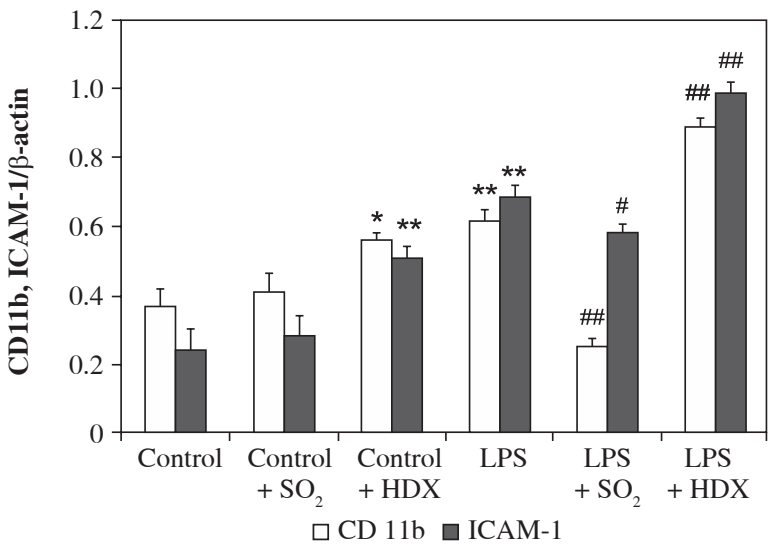

Fig. 6. CD11b and intercellular adhesion molecule-1 (ICAM-1) protein expression by Western blot analysis (A) and densitometric analysis $(\mathbf{B})$ in different groups. Data are presented as the mean $\pm \operatorname{SD}(n=6$ in each group); * $p<0.05$, ** $p<0.01$, compared with control group, \# $p<0.05$, \#\# $p<0.01$, compared with LPS group

A

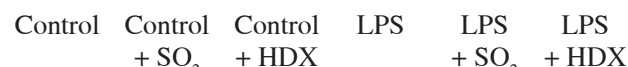

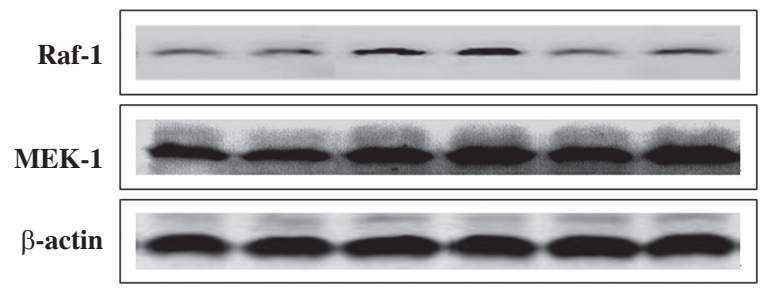

B

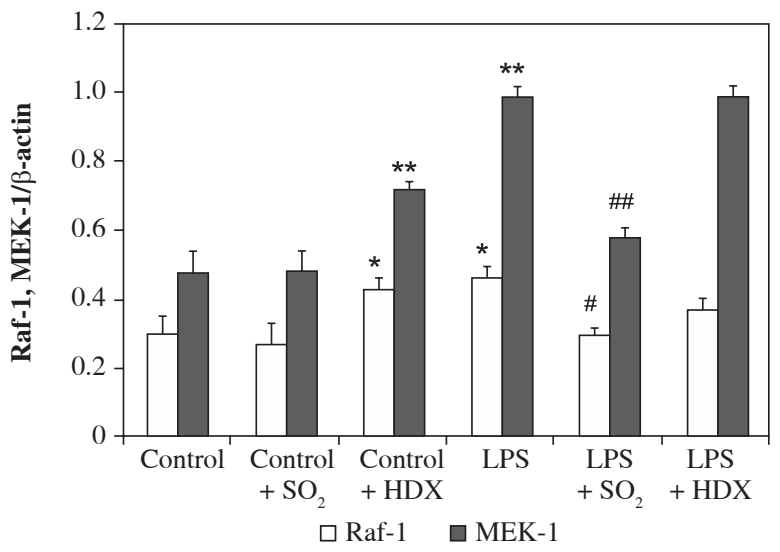

Fig. 7. Raf-1 and MEK-1 protein expression by Western blotting analysis (A) and densitometric analysis (B) in different groups. Data are presented as the mean $\pm \mathrm{SD}$ ( $n=6$ in each group); $* p<0.05$, ** $p<0.01$, compared with control group, $\# p<0.05$, \#\# $p<0.01$, compared with LPS group

A

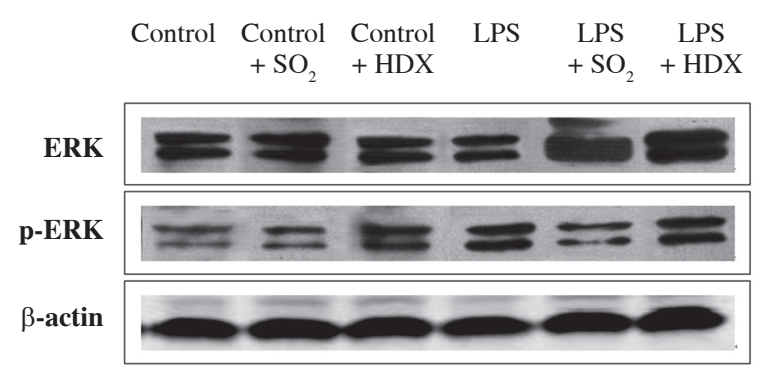

B

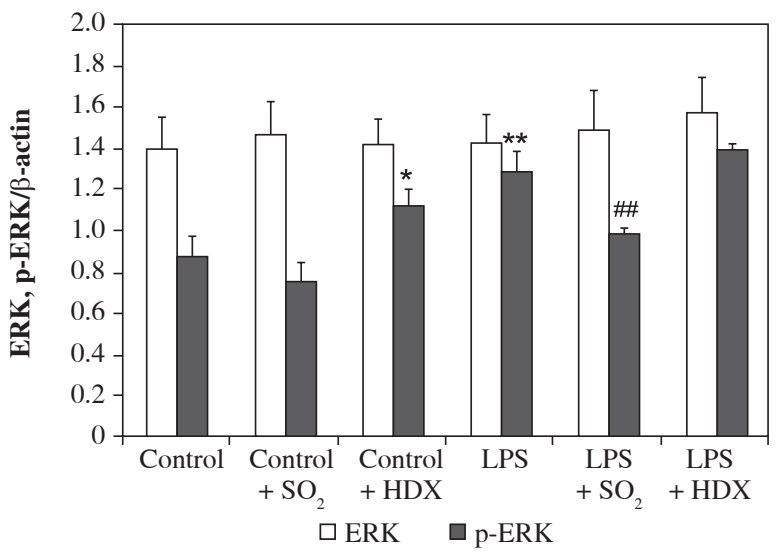

Fig. 8. ERK and p-ERK protein expression by Western blotting analysis $(\mathbf{A})$ and densitometric analysis $(\mathbf{B})$ in different groups. Data are presented as the mean $\pm \mathrm{SD}$ ( $n=6$ in each group); $* p<0.05$, $* * p<0.01$, compared with control group, \#\# $p<0.01$, compared with LPS group 


\section{Determination of PA tension and endothelial function}

PAs were mounted in organ chambers to test the contractile response to PE $\left(10^{-8} \sim 10^{-5} \mathrm{M}\right)$. No differences were found in the responses to this vasoconstrictor among each of the six groups (data not shown).

The endothelial function was tested in endothelium intact PA preconstricted with PE $\left(10^{-6} \mathrm{M}\right)$. Analysis of the concentration-response curves to the endotheliumdependent vasodilator ACh showed no significant change between the control group and the control $+\mathrm{SO}_{2}$ group ( $p>0.05$, Fig. 9). However, the curves significantly shifted to the right both in the LPS group and the control + HDX group of rats when compared with control rats $(p<0.01$, Fig. 9). Moreover, no changes were found in the response to the endothelium-independent vasodilator SNP in PA among the LPS, control + HDX and control groups of rats (data not shown). Analysis of the vessel tension and endothelial function data showed no significant change in the PA endothelium from rats of the control $+\mathrm{SO}_{2}$ group compared with the control group (Figs. 10A and 10B). However, significant structural injury of the PA endothelium was found in the control + HDX group (Fig. 10C) and the LPS group (Fig. 10D) compared to control rats.

Furthermore, the relaxation of PA to ACh showed a significant increase in the LPS $+\mathrm{SO}_{2}$ group, but a decrease in the LPS + HDX group, compared with the LPS group ( $p<0.01$, Fig. 9). Correspondingly, structural changes of the PA endothelium were alleviated in the LPS $+\mathrm{SO}_{2}$ group (Fig. 10E), but aggravated in the LPS + HDX group (Fig. 10F), compared with the control group.

\section{Discussion}

In this study, we demonstrated that $\mathrm{SO}_{2}$ markedly reduced the inflammatory response of ALI induced by LPS, likely by down-regulating Raf-1, MEK-1 and p-ERK during ALI. It is likely that the down-regulated $\mathrm{SO}_{2} / \mathrm{AAT}$ pathway is involved in the underlying mechanism(s) responsible for lung injury induced by LPS. Excessive and uncontrolled inflammatory responses within the lung as well as PAH can complicate the clinical course of ALI induced by endotoxin. Recent research indicates that $\mathrm{SO}_{2}$ may be produced endogenously in vessels and lungs and regulates both vascular reactivity $[21,25]$ and inflammatory responses $[3,19,26]$. $\mathrm{SO}_{2}$ and its hydrated form, sulfite/bisulfite, are generated through the normal processing of sulfur-containing amino acids such as L-cysteine, and AAT can be a key enzyme in controlling the endogenous production of $\mathrm{SO}_{2}$. Exogenous and endogenous $\mathrm{SO}_{2}$ have been shown to exist in plasma and tissue in the form of sulfite-bimane $\left(\mathrm{SO}_{3}{ }^{2-}\right.$ and $\left.\mathrm{HSO}_{3}{ }^{-}\right)$. Thus, the level of sulfite-bimane may represent $\mathrm{SO}_{2}$ content.

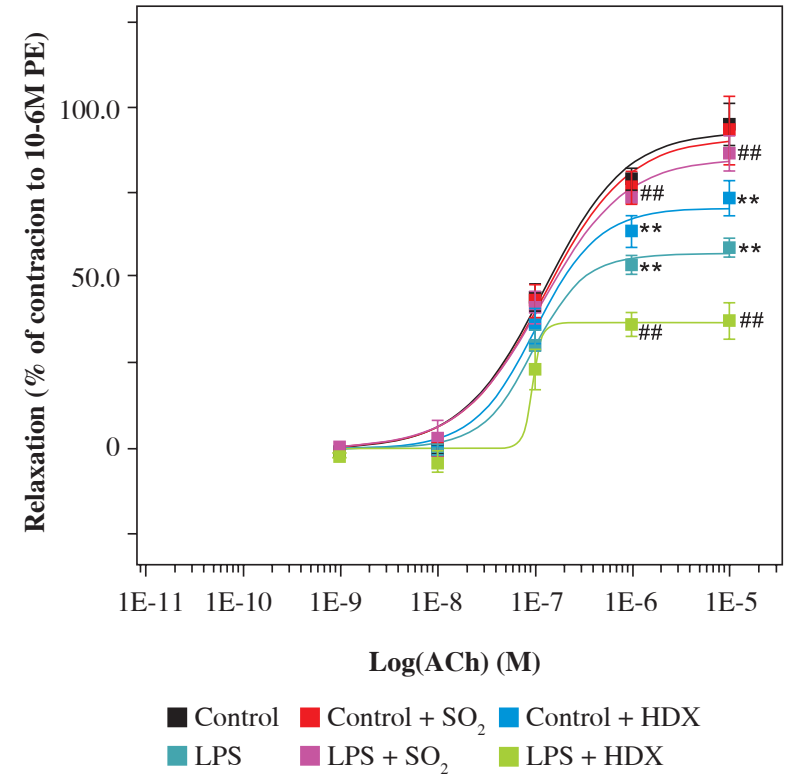

Fig. 9. Endothelium-dependent relaxation responses to acetylcholine $(\mathrm{ACh})$ in PAs. Data are presented as the mean $\pm \mathrm{SD}$ ( $n=8$ in each group); ** $p<0.01$, compared with control group, \#\# $p<0.01$, compared with LPS group (PE - phenylephrine)

In this study, the LPS $+\mathrm{SO}_{2}$ group and LPS + HDX group were included in the experimental design to determine whether the down-regulated $\mathrm{AAT} / \mathrm{SO}_{2}$ pathway is involved in the development of LPS-induced lung injury. The microstructural analysis in the LPS group showed significant lung injury in rats exposed to LPS for $6 \mathrm{~h}$. Interestingly, the $\mathrm{SO}_{2}$ content and AAT activity in lung tissues decreased after LPS stimulation. Under identical LPS exposure conditions, rats of the LPS $+\mathrm{SO}_{2}$ group showed increased $\mathrm{SO}_{2}$ content in lung tissues, whereas the degree of lung injury clearly decreased. However, after treatment with $\mathrm{HDX}$, an AAT inhibitor, the $\mathrm{SO}_{2}$ content in lung tissues decreased, while the lung injury was aggravated significantly. The results suggested that down-regulation of the $\mathrm{SO}_{2} / \mathrm{AAT}$ pathway may be involved in the development of ALI. Interestingly, the results shown in Fig. 5A suggest that HDX produced only a modest effect on AAT activity, while other variables were much more potently affected (e.g., total lung injury). This observation may be due to the non-specific inhibition of AAT by HDX, although it has been used primarily as an AAT inhibitor in other studies [27]. However, since the $\mathrm{SO}_{2}$ concentration was much more potently affected than AAT activity by HDX in our present study (see Fig. 5B), we can reasonably speculate that $\mathrm{HDX}$ inhibited the production of $\mathrm{SO}_{2}$ at least in part through an AAT-independent mechanism. Of course, further study would be needed to discern the 

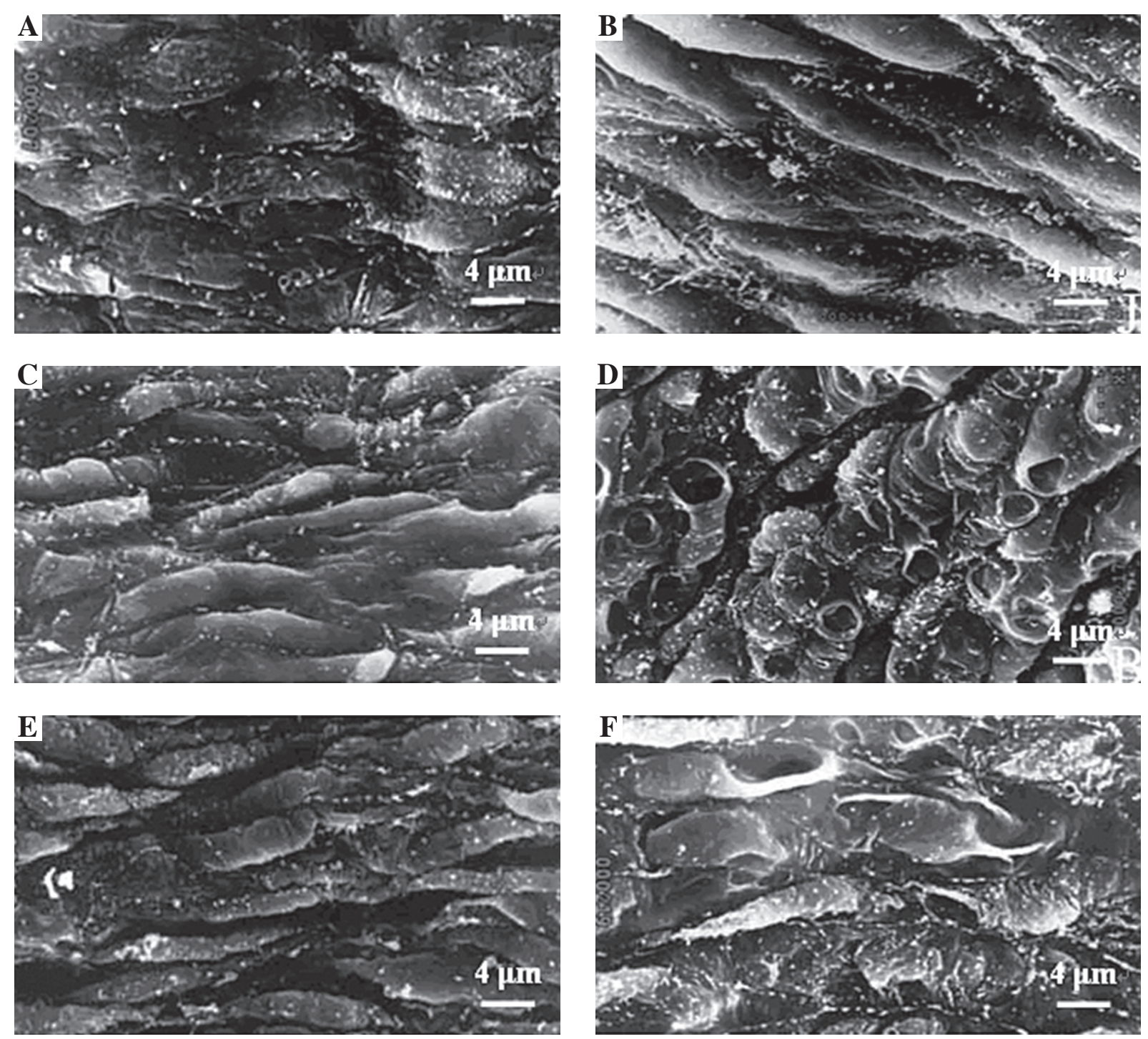

Fig. 10. Scanning electron micrographs of rat PA endothelium in the six groups (1500x). A) Control group; vascular endothelial cells appeared flat. B) Control + sulfur dioxide $\left(\mathrm{SO}_{2}\right)$ group; the plasma membrane of a few endothelial cells was slightly irregular. C) Control + hydroxamate (HDX) group; vascular endothelial cells were swollen. D) LPS group; some vascular endothelial cells were swollen, and some cells were even degenerated. E) LPS + $\mathrm{SO}_{2}$ group; plasma membranes of vascular endothelial cells were relatively even and appeared intact. F) LPS + HDX group: some vascular endothelial cells were swollen, and some cells were even severely degenerated (HDX -)

extent of the contribution of AAT-independent effects to the pattern of injury observed.

PAH is important in the pathogenesis of ALI caused by LPS [28]. In this study, the mean PAP was clearly increased in the LPS-treated groups; meanwhile, $\mathrm{SO}_{2}$ could depress the mean PAP, whereas HDX could significantly augment it in ALI rats. The vessel tension and ultrastructural analyses showed that rats exposed to LPS for $6 \mathrm{~h}$ sustained endothelial injury and exhibited significantly decreased endothelium-dependent relaxation, which could be reversed by $\mathrm{SO}_{2}$ but aggravated by HDX. Our data suggest that $\mathrm{SO}_{2}$ may mitigate $\mathrm{PAH}$-induced by LPS through regulation of the activity in the PA. Nevertheless, the underlying mechanism by which $\mathrm{SO}_{2}$ modulates activity in the PA in ALI warrants further investigation. Interactions between $\mathrm{SO}_{2}$ and other gaseous mediators are potential mechanisms for the attenuation of PAH by $\mathrm{SO}_{2}$. For example, multiple studies have described the functional interaction between $\mathrm{NO}$ and $\mathrm{SO}_{2}$ in spontaneous hypertension and atherosclerosis [18-20], implying that the presence of other gaseous 
molecules in the endothelium influences $\mathrm{SO}_{2}$ availability and/or efficacy. In this study, we observed that LPS treatment precipitated endothelial injury (Figs. 9 and 10). Thus, the altered availability of NO may have influenced the effects of $\mathrm{SO}_{2}$. However, the potential interaction between $\mathrm{SO}_{2}$ and NO in PAH induced by LPS needs to be further studied.

Inflammation is also important in the pathogenesis of lung injury caused by LPS [19]. In this study, MPO activity in the lung, a marker of tissue neutrophil infiltration, was clearly increased in the LPS groups. ICAM-1 and $\mathrm{CD} 11 \mathrm{~b}$ are considered to be pivotal for the accumulation of PMNs in the lung, which is a key inflammatory cell type in the development of ALI [3]. Stimulation of PMNs by injury-related factors, such as LPS, results in the generation of both inflammatory factors (e.g., IL-1 $\beta$, IL-6) and anti-inflammatory factors (e.g., IL-10). In this study, $\mathrm{SO}_{2}$ could reduce the expression of ICAM-1 and $\mathrm{CD} 11 \mathrm{~b}$ and activity of MPO, thereby inhibiting IL-1 $\beta$ and IL-6 but enhancing IL-10 generation in the lung. Meanwhile, HDX could significantly augment the expression of ICAM-1 and CD11b and activity of MPO, consequently enhancing IL-1 $\beta$ and IL- 6 but inhibiting IL-10 generation in the lung.

Our results suggest that $\mathrm{SO}_{2}$ may play a role in regulating the inflammatory response, but this mechanism is unclear and needs further investigation. The mitogen-activated protein kinase (MAPK) pathway is a typical cell signaling pathway that advances inflammatory responses. Here, we examined the expression of Raf-1, MEK-1 and p-ERK/ERK, three main components (arranged in order of activation) in the MAPK pathway, in the lung tissues of LPS-induced ALI rats. Our results indicated that $\mathrm{SO}_{2}$ may inhibit the expression of Raf-1 protein, which is upstream of MEK-1 in the signaling pathway. We also found that the phosphorylation of ERK by MEK-1 was down-regulated, resulting in a decreased $\mathrm{p}$-ERK/ERK ratio. These results suggest that $\mathrm{SO}_{2}$ inhibits IL- $1 \beta$ and IL- 6 but enhances IL-10 generation, likely through the MAPK signaling pathway.

In conclusion, our study suggests that $\mathrm{SO}_{2}$ significantly influences the inflammatory response in association with Raf-1, MEK-1 and p-ERK during ALI induced by LPS. Furthermore, it is likely that the down-regulated $\mathrm{SO}_{2} / \mathrm{AAT}$ pathway is involved in the mechanism responsible for lung injury induced by LPS.

\section{Acknowledgements}

This work was supported by the National Natural Science Foundation of China (No. 30800440 and 81070050), and the Natural Science Foundation of Peking (No. 709203) and Hebei Province (No. H2014206093, H2014206244, H2012206009, C2008001040 and No.
H2016206191), and Hebei Provincial Department of Education (No. ZD2017050).

The authors declare no conflict of interest.

\section{References}

1. Blank R, Napolitano LM (2011): Epidemiology of ARDS and ALI. Crit Care Clin 27: 439-458.

2. Kneyber MC, Markhorst DG (2009): Management of acute lung injury and acute respiratory distress syndrome in children: a different perspective. Crit Care Med 37: 3191-3192; author reply: 3192-3193.

3. Zhou X, Dai Q, Huang X (2012): Neutrophils in acute lung injury. Frontiers in bioscience : a journal and virtual library 17: 2278-2283.

4. Fujishima S, Aikawa N (1995): Neutrophil-mediated tissue injury and its modulation. Intensive Care Med 21: 277-285.

5. Mecklenburgh K, Murray J, Brazil T, et al. (1999): Role of neutrophil apoptosis in the resolution of pulmonary inflammation. Monaldi Arch Chest Dis 54: 345-349.

6. Gao XP, Liu Q, Broman M, et al. (2005): Inactivation of $\mathrm{CD} 11 \mathrm{~b}$ in a mouse transgenic model protects against sepsis-induced lung PMN infiltration and vascular injury. Physiol Genomics 21: 230-242.

7. Ramos-Mozo P, Madrigal-Matute J, Martinez-Pinna R, et al. (2011): Proteomic analysis of polymorphonuclear neutrophils identifies catalase as a novel biomarker of abdominal aortic aneurysm: potential implication of oxidative stress in abdominal aortic aneurysm progression. Arterioscler Thromb Vasc Biol 31: 3011-3019.

8. Arias-Diaz J, Villa N, Hernandez J, et al. (1997): Carbon monoxide contributes to the cytokine-induced inhibition of surfactant synthesis by human type II pneumocytes. Arch Surg 132: 1352-1360; discussion: 1360-1351.

9. Connelly L, Palacios-Callender M, Ameixa C, et al. (2001): Biphasic regulation of NF-kappa $\mathrm{B}$ activity underlies the proand anti-inflammatory actions of nitric oxide. J Immunol 166: 3873-3881.

10. Huang XL, Zhou XH, Zhou JL, et al. (2009): Role of polymorphonuclear neutrophil in exogenous hydrogen sulfide attenuating endotoxin-induced acute lung injury. Sheng Li Xue Bao 61: 356-360.

11. Morisaki H, Katayama T, Kotake Y, et al. (2002): Carbon monoxide modulates endotoxin-induced microvascular leukocyte adhesion through platelet-dependent mechanisms. Anesthesiology 97: 701-709.

12. Hong YC, Lee JT, Kim H, et al. (2002): Effects of air pollutants on acute stroke mortality. Environ Health Perspect 110: 187-191.

13. Meng Z (2003): Oxidative damage of sulfur dioxide on various organs of mice: sulfur dioxide is a systemic oxidative damage agent. Inhal Toxicol 15: 181-195.

14. Meng Z, Liu Y (2007): Cell morphological ultrastructural changes in various organs from mice exposed by inhalation to sulfur dioxide. Inhal Toxicol 19: 543-551.

15. Meng Z, Nie A (2005): Effects of sodium metabisulfite on potassium currents in acutely isolated CA1 pyramidal neurons of rat hippocampus. Food Chem Toxicol 43: 225-232. 
16. Meng Z, Zhang B, Ruan A, et al. (2002): Micronuclei induced by sulfur dioxide inhalation in mouse bone-marrow cells in vivo. Inhal Toxicol 14: 303-309.

17. Du SX, Jin HF, Bu DF, et al. (2008): Endogenously generated sulfur dioxide and its vasorelaxant effect in rats. Acta Pharmacol Sin 29: 923-930.

18. Luo L, Chen S, Jin H, et al. (2011): Endogenous generation of sulfur dioxide in rat tissues. Biochem Biophys Res Commun 415: 61-67.

19. Ma HJ, Huang XL, Liu Y, et al. (2012): Sulfur dioxide attenuates LPS-induced acute lung injury via enhancing polymorphonuclear neutrophil apoptosis. Acta Pharmacol Sin 33: 983-990.

20. Lu W, Sun Y, Tang C, et al. (2012): Sulfur dioxide derivatives improve the vasorelaxation in the spontaneously hypertensive rat by enhancing the vasorelaxant response to nitric oxide. Exp Biol Med (Maywood) 237: 867-872.

21. Zhang Q, Meng Z (2009): The vasodilator mechanism of sulfur dioxide on isolated aortic rings of rats: Involvement of the $\mathrm{K}+$ and Ca2+ channels. Eur J Pharmacol 602: 117-123.

22. Kristof AS, Goldberg P, Laubach V, et al. (1998): Role of inducible nitric oxide synthase in endotoxin-induced acute lung injury. Am J Respir Crit Care Med 158: 1883-1889.

23. Labarca C, Paigen K (1980): A simple, rapid, and sensitive DNA assay procedure. Anal Biochem 102: 344-352.

24. Mitsuhashi H, Ikeuchi H, Yamashita S, et al. (2004): Increased levels of serum sulfite in patients with acute pneumonia. Shock 21: 99-102.

25. Wang YK, Ren AJ, Yang XQ, et al. (2009): Sulfur dioxide relaxes rat aorta by endothelium-dependent and -independent mechanisms. Physiol Res 58: 521-527.

26. Huang XL, Zhou JL, Zhou XH, et al. (2009): Ameliorative effects of exogenous sulfur dioxide on lipopolysaccharide-induced acute lung injury in rats. Sheng Li Xue Bao 61: 499503.

27. Sun Y, Tian Y, Prabha M, et al. (2010): Effects of sulfur dioxide on hypoxic pulmonary vascular structural remodeling. Lab Invest 90: 68-82.

28. Spapen H, Vincken W (1992): Pulmonary arterial hypertension in sepsis and the adult respiratory distress syndrome. Acta Clin Belg 47: 30-41. 